\title{
Electromagnetic and structural global model of the TF magnet system in ASDEX Upgrade
}

\author{
I. Zammuto, B. Streibl, L. Giannone, A. Herrmann, A. Kallenbach, V. Mertens, ASDEX \\ Upgrade Team
}

Max-Planck-Institut für Plasmaphysik, EURATOM Association, D-85740 Garching, Germany

The enhancements carried out in the tokamak ASDEX Upgrade (AUG) are oriented towards the preparation of the future physics-related activities of ITER and DEMO. To address the main ITER issues, plasma configurations with a wider operational limit (e.g. higher triangularity) are planned for the future experimental campaigns in AUG. To evaluate the mechanical impact on the toroidal field (TF) magnet system a combined electromagnetic and structural finite element model was set up.

At first extensive benchmarks of the models are carried out against the AUG reference design configurations with respect to stress [1-3] and lateral displacement measurements. The numerical model was then applied to a set of actual high triangularity (HT) configurations generated by a more favorable PF current distribution made possible by an extension of the power supply system. The resulting change of the poloidal flux pattern and the lateral force distribution has consequences for the coil shear stress and vault stability. Both aspects are monitored by a safety system measuring the PF flux placed on top and bottom of the outer surface of two TF coils between vault and TOS. The range of the new HT configurations has induced a modification of the flux pattern, so that an adaptation of the safety system is required to protect the TF system. Following the same criteria of the old safety system, a new set up of virtual coils will be integrated in the control system of AUG. The motivation of this new set up will be discussed in this paper.

Keywords: ASDEX Upgrade, ANSYS, Electromagnetic modeling, Toroidal field coil.

\section{Introduction}

This paper illustrates the numerical analyses carried out to verify the load carrying capability of the toroidal magnetic field (TF) magnet in ASDEX Upgrade (AUG).

The main purpose of the analysis presented is to demonstrate that the mechanical components of the TF magnet are able to withstand the loads induced by the high triangularity configurations which are planned for the near future of the exploitation of AUG.

The analysis has been carried out using the commercial finite element code ANSYS. Both the electromagnetic (EM) and the structural models require only two of the 16 sectors AUG for describing the complete operational demands (self weight, TF on and plasma operation).

A benchmark of the FE model against the design parameters has been carried out: an extensive comparison in terms of design forces, stresses and deformations has been made and critically analyzed. In a second step, thanks to the different diagnostics which are monitoring the TF coil (TFC) behavior, a benchmark against the TF displacements has been done to demonstrate the reliability of the FE model.

Finally, in the benchmarked model, an improvement of the safety system has been proposed according to the change of the poloidal field pattern.

\section{Basic design information}

During normal operating conditions, the TF magnet system has to withstand the EM loads arising from the interaction of its conductor current with both the toroidal and poloidal fields.

The interaction with the toroidal field gives rise to the force distribution acting in the plane of the each coil. Due to the "constant tension" coil shape the turns are mainly loaded by circumferential stresses. The 
straight inner legs of all 16 coils form a vault to balance the centering force resulting from the pressure component. The centering force per coil has a maximum value of about $16 \mathrm{MN}(\mathrm{B} 0=3.9 \mathrm{~T})$.

The poloidal field, required for balancing and shaping the plasma, gives rise to an out-of-plane load component due to the interaction with the TF magnet current. This load does not produce a net moment around the vertical axis of the machine center but tries to twist each TFC and to turn it around the radialaxis. A stiff stainless steel structure, the so called Turn Over Structure - TOS, counteracts mainly the outof-plane forces. A minor part is in addition taken up by friction forces in the vaulted inner coil legs. There remains therefore only a small non-supported fraction of the coil circumference that has to take up the lateral forces by bending.

\section{Modeling}

The TOS consists of 8 big casings flanged in the mid plane and 8 small casings flanged laterally with the adjacent big casings (Fig. 1). Symmetry conditions call for a FE model with two TFCs and their TOS sectors, in particular:

- 1 complete TFC with 2 pancakes ( $2 * 12$ turns), with its grounding insulation;

- 2 half TFCs, split in their mid-plane, hence with 1 pancake each (12 turns), with its grounding insulation;

- 1 big casing of the TOS;

- 1 small casing of the TOS, consisting of an upper and a lower part due to the opening of the mid-plane port.

More details on the TF magnet system and its supporting structure are available in [1-3].

The TFC interfaces have been meshed using the ANSYS technology contact elements. Such interfaces are implemented at:

- The vault epoxy bladders between adjacent TFCs;

- The lateral epoxy bladders placed in the outboard between TF-coil and TOS and

- The frictional weight support pad on the bottom of the coil, sliding on the TOS during ramp-up.

These interfaces are modeled by a surface-to-surface contact pair, taking into account the respective friction coefficients.
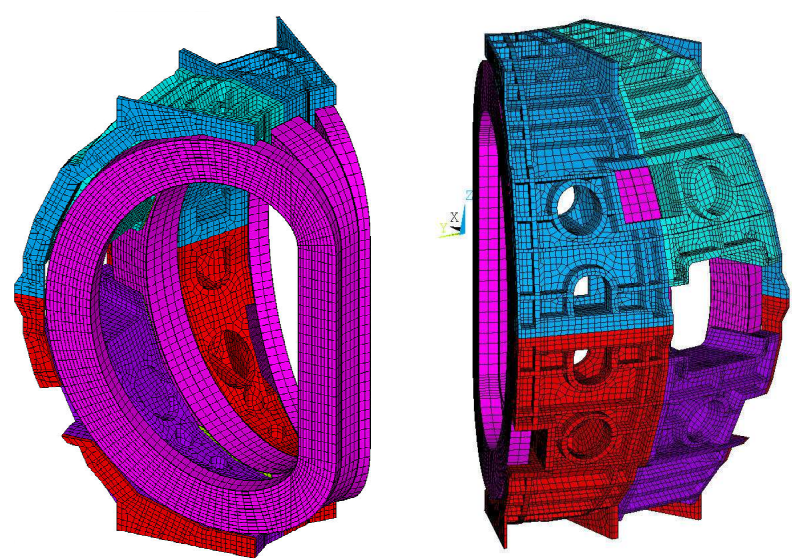

Fig. 1: Overview of the AUG structural model.

\section{Comparison with the reference case}

The reference case is the scenario originally used for designing the TFCs. Each plasma configuration has one reference case. In this work only the single null (SN) configuration will be shown, for reference. 
The main parameters describing this configuration are reported in [2]. It is worth mentioning that, compared to the configurations used for the TFC design, nowadays two additional PF coils, the $\mathrm{OH} 2$ coils, improve the plasma shaping.

\subsection{In-plane forces}

A total centering force of $16 \mathrm{MN}$ per each TFC is results from the EM model, compared to a value of 15.7 $\mathrm{MN}$ as originally estimated in the design phase. In the following plots of the force distribution the abscissa spans the TF poloidal profile, starting from the mid plane of the straight leg and running along the TF current direction, passing through the upper straight leg, upper transition, outboard, lower transition and lower straight leg. In Fig. 2 the average in-plane forces per unit length $[\mathrm{MN} / \mathrm{m}]$ along the TF profile are shown. The maximum value is on the straight leg, where the centripetal line load reaches $8 \mathrm{MN} / \mathrm{m}$. The red vertical line represents the mid plane in the outboard. The pink lines define the starting and ending points of the TF transition regions, which are the most critical parts for bending stresses arising during plasma operation.

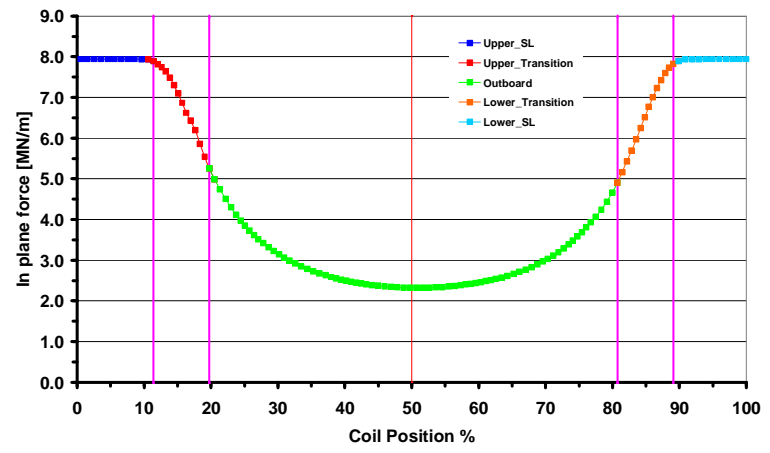

Fig. 2: In-plane forces per unit length $[\mathrm{MN} / \mathrm{m}]$ along the circumferential TF profile for the $\mathrm{SN}$ reference case. The pink lines are defining the upper and lower transition regions of the TF. The red line indicates the mid plane in the TF outboard.

\subsection{Out-of-plane forces}

In Fig. 3 the average out-of-plane forces per unit length [MN/m] along the poloidal TF profile are reported. The maximum lateral line load, of about $1 \mathrm{MN} / \mathrm{m}$ occurs in the upper transition area, after the straight leg. The red and pink lines in Fig. 2 correspond to those of Fig. 1. The vertical green lines are identifying the position of the pick up coils used as safety system. It can be already observed that the outof-plane forces peaks are well within the measuring areas.

For the upper half of the TFC results from the EM model:

- $\mathrm{F}_{\text {radial }}=8.02 \mathrm{MN}$ (inward, half of the centering force)

- $\mathrm{F}_{\text {lateral }}=0.25 \mathrm{MN}$

- $\mathrm{F}_{\text {vertical }}=8.31 \mathrm{MN}$

The values of these forces agree reasonably with the original design data.

On the other hand, the peak shear stresses observed in the structural model results quite low compared with the original expectations of the design phase: $11.5 \mathrm{MPa}$ against $20 \mathrm{MPa}$, respectively. The simplified modeling approach of the original design model might explain the discrepancy. The shear stresses now observed with the more detailed ANSYS model are thus welcome for extending the operational range of AUG. 


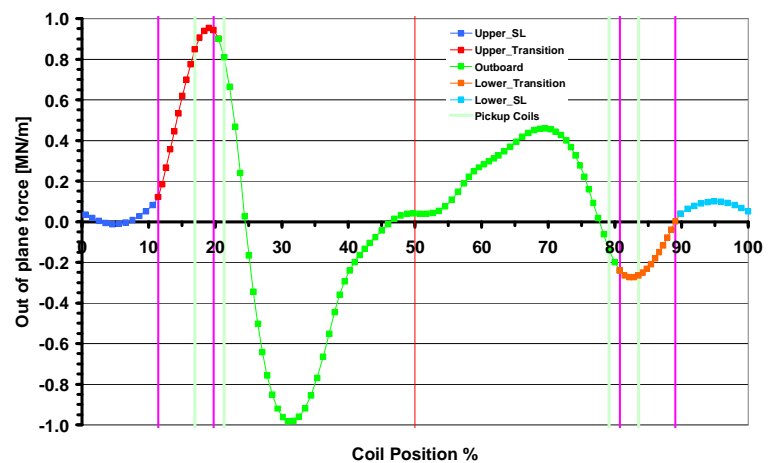

Fig. 3: Out-of-plane forces per unit length $[\mathrm{MN} / \mathrm{m}]$ along the circumferential TF profile under plasma operation for the SN reference case. The vertical pink lines are defining the upper and lower transitions regions of the TF. The vertical green lines highlight the regions where the pick up coils are placed.

\section{Benchmark against displacement measures}

Displacement sensors monitoring the movement of the TFCs are placed nearby the transitions (on the lower and upper part) of all coils. The aim of these sensors is to check possible anomalous TF in- and outof-plane movements that can jeopardize the structure, i.e. a wrong engagement of the coils in the vault. These sensors are monitoring lateral and radial movements on both positions, and vertical movements only on the upper part.

The sensors placed in the TF lower transition region are directly connected to the non-deformed PF supporting structure, thus directly measuring the radial and lateral displacements in almost absolute terms.

The sensors on the TFC upper transition region are measuring less directly radial, lateral and vertical movements of the TFC relative to the TOS tip, which is also deformed by lateral forces. The benchmark of the ANSYS structural model will be carried out comparing the calculated displacements with the measurements of the lower sensors.

In any case these sensors cannot discriminate between the displacements due to the magnetic forces from those due the thermal expansion induced by the Joule heating in the coil itself. Nevertheless they can provide an important information for the modeling of the mechanical structure: the dimension of the lateral gap between the epoxy filled bladders and the TF lateral surfaces.

To benchmark the model against the TFC displacement the shots \# 27327 is considered. In Fig. 4 the measured and calculated displacements are reported. The pink dashed line, the radial displacements, expression of the in-plane forces, are present as soon as the TFs are switched on (at $t=-6.5 \mathrm{~s})$. The negative sign indicates that the TF is pushed inwards, towards the center of the machine. The radial displacement reaches its maximum value in correspondence of the maximum TF current. Then, the heating up of the TF generates its thermal expansion, which is additionally registered by the sensors. Therefore, even beyond end of the plasma discharge, the TFC is gradually moving outward.

In Fig. 4 the lateral displacement is indicated by a blue dashed line. The presence of a gap between TF and bladder plates is evident: During ramp up of the $\mathrm{OH}$ transformer (from $\mathrm{t}=-4 \mathrm{~s}$ onwards), stray fields cause a positive excursion of about $1 / 10 \mathrm{~mm}$ until the coil gets contact with the bladders. During plasma ramp up ( $\mathrm{t}>0 \mathrm{~s}$ ) stronger lateral forces are pushing the coil in opposite (negative) direction until the contact with the other adjacent bladders is reached. After plasma are switch-off, the TFC is rapidly moving back and forth, from one side to the opposite one before gradually returning in its resting position. 


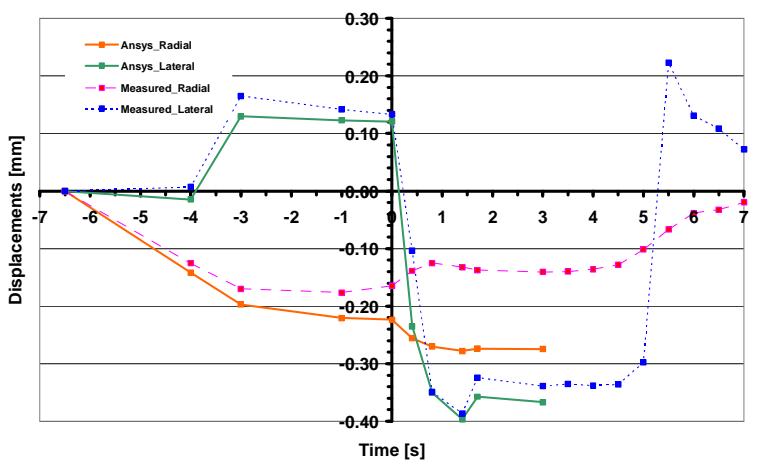

Fig. 4: Displacements [mm] for the shot \# 27327: red and green solid lines are relative to the computed radial and lateral displacements, respectively. Pink and blue dashed lines are respectively for the measured radial and lateral displacements (both without thermal expansion).

To introduce the lateral TF displacement as shown by the measurements, a gap of $0.12 \mathrm{~mm}$ is modeled between TF and bladder surfaces. With this adjustment, a good agreement between the computed and the measured displacements is confirmed by Fig. 4. There is almost an overlap between the dashed and solid lines representing the measured and computed displacements, respectively.

\section{Recent high triangularity discharges}

After the extensive benchmark of the FE model with the design cases and the available measurements, the evaluation of forces and stresses in the TFC for the present magnetic field configuration has been performed. In this case also the $\mathrm{OH} 2$ coils contribute to the plasma shaping. The change of the poloidal field configuration affects only the distribution of the out-of-plane forces. In Fig. 5 the out-of-plane forces for typical plasma shots, normalized to $1 \mathrm{MA}$ plasma current are summarized. Fig. 5 shows that the maximum of the out-of-plane forces coincides no longer with the pick up coil positions. The contribution of the $\mathrm{OH} 2$ coils for plasma shaping shifts the maximum throughout closer towards the vault.

It has to be pointed out that the stress level in the present shots is well below the allowable limits established for AUG. In particular, the main constrain is given by the allowable shear stress of $20 \mathrm{MPa}$ in the turn insulation. However, from the computations for the discharges of Fig. 5 results only a value of about $10 \mathrm{MPa}$ for a large plasma current (1.2 MA).

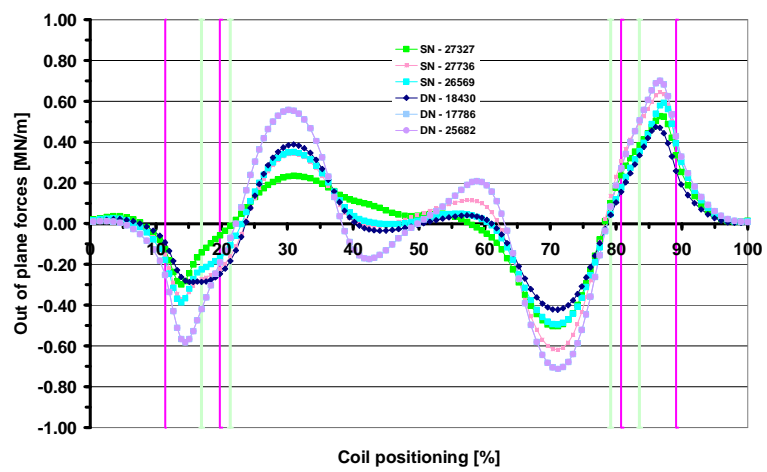

Fig. 5: Out-of-plane forces per unit length $[\mathrm{MN} / \mathrm{m}]$ along the circumferential TF profile under different plasma operation. The pink lines are defining the upper and lower transitions regions of the TF. The green lines highlight the regions where the pickup coils are placed.

\section{Safety system}


In two TFCs, pick-up coils are mounted on the external surfaces of the upper and lower transition regions. These sensors have to control the stability of the TF vault in two different aspects: the friction coefficient in the wedge which counteracts the turn over moment and the shear stress in the TF insulations. The first one is an expression of the capability of the TF wedge to carry the turn over moment, below the allowable friction coefficient of $\mu_{\text {Ref }}=0.2$. The purpose of the last one is to avoid large strains in the layers of the turn insulation for maintaining the integrity of the winding package. The limit on the shear stress of insulations is fixed to $20 \mathrm{MPa}$. The combined information of the upper and lower pickup coils provides the following quantities:

- The ratio between the actual friction coefficient during a discharge and the allowable friction coefficient $\left(\mu_{\text {Ref }}\right)$ represented by a reference case, according to the following expressions:

$$
\mu / \mu_{\text {Ref }}=\left(\phi_{b}+\phi_{t}\right) /\left(\phi_{b}+\phi_{t}\right)_{\text {Ref }} * B_{\text {oRef }} / B_{o}
$$

- The ratio between the shear stress of a particular discharge and the maximum shear stress evaluated for a typical reference case, according to the following expression:

$$
\tau / \tau_{\text {Ref }}=\left(\phi_{b}-\phi_{t}\right) /\left(\phi_{b}-\phi_{t}\right)_{\operatorname{Ref}} * B_{o} / B_{o R e f}
$$

where $\mu$ and $\tau$ are the friction coefficient and the shear stress of the shot where the measures are taken, respectively; $\phi_{\mathrm{b}}$ and $\phi_{\mathrm{t}}$ are the magnetic fluxes for the bottom and lower pickup coils, respectively; $\mathrm{B}_{\mathrm{o}}$ is the toroidal magnetic field on the plasma axis $\left(\mathrm{R}_{\mathrm{o}}=1.65 \mathrm{~m}\right)$. All parameters with the subscript 'Ref' are referred to the allowable values of the corresponding reference case. For each plasma configuration, SN and $\mathrm{DN}$, different reference values are defined.

The correct positioning of the sensors with respect to the maximum of the lateral line load is crucial evaluation for the relations (1) and (2). However, as seen in Fig. 5, the measured magnetic fluxes are no longer corresponding to the place where the maximum forces exerted on the TFCs arise. In addition, a careful evaluation of the measured flux demonstrates that a recalibration of such pickup coils is ineffective, due to the missing linearity of the measured fluxes with the forces. So, an improvement of the safety system is required to monitor the TF magnet. Virtual pickup coils will be implemented in the control system of AUG. The support of the FE models, both electromagnetic and structural, is required to define the best position of the virtual coils and to provide the reference values reported in (1) and (2). Corresponding to the shifted maximum of the out-of-plane forces, the locations of four pickup coils in the upper and in the lower transition regions will be optimized. The improved safety system will be operational for the next experimental campaign.

\section{Summary and conclusion}

The results of the extended numerical model provide an additional structural safety margin for the AUG TF magnet relative to the design case. This permit to extend the operational range of AUG to the envisaged more demanding high triangularity discharges. For future significant changes of the PF configuration there exist now a versatile FE model for verifying the mechanical soundness of the TFC prior to execution of the discharge.

The new TF-safety system with virtual pickup coils provides now high flexibility with respect to configuration changes. For the next experimental campaign, the new safety system will be implemented and adjusted according to the described change of the poloidal magnetic field configuration.

\section{References}

[1] ASDEX Upgrade Project Proposal - Phase II , ASDEX Upgrade Project Team, IPP Report 1/217 May 1983.

[2] O. Jandl, E. Springmann, B. Streibl, Comparison of pumping limiter, double null and single null divertor condition for ASDEX Upgrade toroidal field magnet, 8th International Conference on Magnet Thecnology, Jounrnal de Physycs Supplement, September 1983.

[3] S. Mukherjee, E. Harmeyer, B. Streibl, 3D structural analysis of the Toroidal Field (TF) magnet for ASDEX Upgrade, 10th International Conference on Magnet Thecnology, Boston, September 1987, paper CR-8.

[4] L. Giannone, Tipping force monitoring of the ASDEX Upgrade Toroidal Field coil, internal report. 